EPJ Web of Conferences 43, 05006 (2013)

DOI: $10.1051 /$ epjconf/20134305006

(C) Owned by the authors, published by EDP Sciences, 2013

\title{
The DB gap and a new class of pulsating white dwarfs
}

\author{
H. Shibahashi ${ }^{\mathrm{a}}$ \\ Department of Astronomy, University of Tokyo, Tokyo 113-0033, Japan
}

\begin{abstract}
The recent systematic surveys providing enormously massive datasets of white dwarfs show that there is still a deficit of a factor of 2.5 in the DA/non-DA ratio within the temperature range of $30000 \mathrm{~K}<T_{\text {eff }}<45000 \mathrm{~K}$, which has been regarded as the "DB gap" meaning a range with almost no helium atmosphere white dwarfs. Since all white dwarfs have to evolve through this temperature range along almost the identical sequence on the color-magnitude diagram, this implies that most of the helium atmosphere DO stars once evolve into hydrogen atmosphere hot DA stars in the temperature range of the DB gap and then back to helium atmosphere DB stars. Possible scenarios for this chameleon-like disguises of white dwarfs with helium dominant atmospheres are described and a new class of pulsating white dwarfs, named the hot-DAV stars, is predicted from these scenarios. One pulsating DA white dwarf, being consistent with the prediction, has been discovered indeed.
\end{abstract}

\section{INTRODUCTION}

Spectroscopic observations indicate that most of the white dwarfs show only either hydrogen absorption lines or helium lines. This purity is thought to be caused by the high surface gravity, which gravitationally separates the envelope atmosphere so that heavy elements are on the bottom and lighter ones on top. The majority of white dwarfs are then divided in terms of spectroscopic features into two major subgroups: those showing hydrogen Balmer lines only and those showing helium lines only. The former, constituting $\sim 85$ per cent of all white dwarfs, are classified as DA white dwarfs by analogy with main sequence A stars whose visible spectra are dominated by Balmer absorption lines of hydrogen. The latter are classified as DB white dwarfs, and a smaller fraction as DO white dwarfs by analogy with the main sequence $\mathrm{O}$ and $\mathrm{B}$ stars whose visible spectra show strong absorption lines of ionized and neutral helium, respectively.

Since the envelope mass is so small, the influence of the surface composition on the sequence of white dwarfs in the color-magnitude diagram cannot be seen. Hence, the sequence of white dwarfs is almost identical both for DA white dwarfs and DO and DB white dwarfs. Since there is no longer any heating source in white dwarfs, this well-defined sequence of white dwarfs on the color-magnitude diagram represents a cooling sequence. The DA white dwarfs are found across a wide range of surface temperatures, from $T_{\text {eff }}=100000 \mathrm{~K}$ at the hot end down to $T_{\text {eff }}=6300 \mathrm{~K}$. Unlike the DA white dwarfs, DO stars are found only in the restricted temperature range $80000 \mathrm{~K} \geq T_{\text {eff }} \geq 45000 \mathrm{~K}$, and DB stars mainly in the range $30000 \mathrm{~K} \geq T_{\text {eff }} \geq 12000 \mathrm{~K}$. Prior to the systematic surveys such as the Sloan Digital Sky Survey (SDSS) providing enormously massive datasets ([2], [7]), white dwarfs with no helium atmosphere were known between these two temperature range, although the temperature range between $45000 \mathrm{~K}$ and $30000 \mathrm{~K}$ spans from the DO to DB stars. This temperature range became for simplicity known only as the "DB gap", meaning a range with almost no helium atmosphere white dwarfs ([10], [11]). This implies that, since all white dwarfs have to evolve through this temperature range as they

\footnotetext{
a e-mail: shibahashi@astron.s.u-tokyo.ac.jp
}

This is an Open Access article distributed under the terms of the Creative Commons Attribution License 2.0, which permits unrestricted use, distribution, and reproduction in any medium, provided the original work is properly cited. 
radiate away their residual heat, somehow helium atmosphere DO stars evolve into hydrogen atmosphere hot DA stars, then back to helium atmosphere DB stars. Now, the SDSS discovered more than twenty DB white dwarfs within the temperature range between $45000 \mathrm{~K}$ and $30000 \mathrm{~K}$ ([3], [5]). In this sense, this temperature range is strictly speaking no longer a gap. However, it is found that there is still a deficit of a factor of 2.5 in the DA/non-DA ratio within this temperature range ([3]). Hence the puzzle to be solved still remains: why such chameleon-like disguises, from helium atmosphere to hydrogen atmosphere and again back to helium atmosphere, are seen?

\section{PLAUSIBLE SCENARIOS FOR SPECTRAL SVOLUTION AND THE DB GAP}

In the case of white dwarfs with helium atmosphere, hydrogen does not necessarily have to be completely absent. Small amount of contamination would not be ruled out even if hydrogen lines are not detectable. How much hydrogen can be still retained in the envelope of DO or DB white dwarfs is quite uncertain, but presumably the upper limit is less than $10^{-14} M_{\odot}$. If hydrogen more than $10^{-14} M_{\odot}$ is left after the post-AGB phase, the star shall be regarded as a DA white dwarf. ${ }^{1}$

The presence of the DB gap implies that white dwarfs with helium dominated atmosphere including a tiny amount of residual hydrogen may change their spectroscopic type as they cool down, probably due to the interplay of diffusion and convection, though the chemical evolution is quite complicated and not completely understood.

Fontaine \& Wesemael ([4]) explained the DB gap as a consequence of the evolution of almost all white dwarfs from planetary nebulae nuclei. They supposed that a slow rise of hydrogen to the surface, as heavier nuclei sink in the strong gravitational field, eventually makes helium-rich white dwarfs appear as DA stars at the blue edge of the DB gap at about $45000 \mathrm{~K}$.

Shibahashi ([12]) proposed a different model for the onset of the blue edge of the DB gap. At temperatures where the atmosphere is convective $\left(T_{\text {eff }}>45000 \mathrm{~K}\right)$ due to the second helium ionization, if the amount of hydrogen is sufficiently small, turbulence thoroughly mixes the hydrogen in the atmosphere with helium and leads to an apparent helium star, while stars having too much hydrogen to mix completely remain DA white dwarfs. But in the temperature range along the cooling sequence from $45000 \mathrm{~K}$ to $30000 \mathrm{~K}$, known as the "DB gap", the second helium ionization zone is too deep to mix hydrogen, and the little remaining hydrogen floats to the surface, hiding the helium layer beneath it ([4], [12], [13]).

The red edge of the DB gap at $T_{\text {eff }} \sim 30000 \mathrm{~K}$, is explained as a natural consequence of the onset of a significant convection zone at the temperature where the first helium ionization zone coincides with the upper atmosphere, thus creating turbulence to mix the small amount of residual hydrogen into a deeper sea of helium, so the star then reappears as a DB white dwarf ([4], [12], [13]). Stars with hydrogen more than $10^{-14} M_{\odot}$ have too much hydrogen to be well mixed, and they remain DA stars.

\section{PREDICTION OF A NEW TYPE OF PULSATING WHITE DWARFS}

According to the above scenarios, the photospheric level of a white dwarf in the DB gap is convectively stable; $\mathrm{d} \ln \rho / \mathrm{d} \ln p>\gamma^{-1}$, where $\rho$ and $p$ denote the density and the pressure, respectively, and $\gamma$ is the adiabatic exponent defined by $\gamma \equiv(\partial \ln p / \partial \ln \rho)_{S}$, where $S$ means the entropy. Then $\mathrm{d} \ln \rho / \mathrm{d} \ln p=$ $1-\mathrm{d} \ln T / \mathrm{d} \ln p+\mathrm{d} \ln \mu / \mathrm{d} \ln p>\gamma^{-1}$, where $T$ and $\mu$ denote the temperature and the mean molecular weight, respectively.

The radiative temperature gradient, $\mathrm{d} \ln T / \mathrm{d} \ln p$, of the atmosphere gradually becomes steeper when the star cools down, and the surface atmosphere will eventually come to be convectively unstable to mix

\footnotetext{
1 The mass of the overlying hydrogen-rich layer is supposed to be less than approximately $10^{-4} M_{\odot}$. Otherwise the lower portion of the envelope would still be hot enough for nuclear reactions to keep going there.
} 


\section{Ageing Low Mass Stars: From Red Giants to White Dwarfs}

hydrogen with helium. However, since the stars being still in the DB gap but located near the cool end of the DB gap are at the transition phase from the radiative atmosphere to the convective one, they are expected to have a superadiabatic layer (i.e., $\mathrm{d} \ln T / \mathrm{d} \ln p-1+\gamma^{-1}>0$ ), which is nonetheless still convectively stable (i.e., $\mathrm{d} \ln \mu / \mathrm{d} \ln p>\mathrm{d} \ln T / \mathrm{d} \ln p-1+\gamma^{-1}$ ) due to the fact the hotter but heavier element (helium) is on the bottom and the cooler but lighter element (hydrogen) is on the top.

Though such layers are convectively (dynamically) stable, pulsational instability of nonradial g-modes could occur. The physical cause of this instability is that the radiative heat exchange brings about an asymmetry in the oscillatory motion in such a way that an oscillatory element overshoots its equilibrium position with an increasing velocity. The possibility of such instability in a white dwarf was discussed by Baglin more than thirty years ago ([1]). However, the dissipation in the other subadiabatic layers might be strong enough to damp the global oscillation as a whole. It was shown later that the acoustic and gravity waves which are responsible for nonradial oscillations are propagative in the radial direction only in restricted regions (see, e.g., [14]). If the g-modes are well trapped so that their amplitudes are large only in the superadiabatic, convectively stable layer with varying chemical composition, they are likely to be self-excited by the forementioned mechanism. This condition is satisfied in the case of high-degree g-modes. Since the heat exchange in the superadiabatic, convectively stable layer becomes efficient in the case of short wavelength, high-degree g-modes are also favorable for excitation. Hence the DA white dwarfs near the cool end of the DB gap must be definitely pulsationally unstable for high-degree g-modes.

Generally speaking, the wave trapping tends to become less efficient with the decrease of the degree $\ell$. However, if the temperature gradient in the bottom layer is really almost the adiabatic one, the low-degree g-modes might also be well-trapped in the superadiabatic layer with varying chemical composition. Whether the temperature gradient is really close to the adiabatic one, or in other words whether the Brunt-Väisälä frequency $N$ is quite low, is a delicate issue, and definitely a careful modeling is necessary. As far as the condition is realized, low-degree modes with $\ell \leq 4$, which may be detectable even though a distant star is a point source, are also excited. This implies that we can confirm the present hypothesis for explanation about the cause of the DB gap, by looking for the predicted pulsating DA white dwarfs near the cool end of the DB gap.

\section{SEARCH FOR THE NEW CLASS OF HOT-DAV STARS}

We started off a search for these predicted new-type of variable white dwarfs that we call the hot-DAV stars. We used as our source for target selection the catalogue of spectroscopically identified white dwarf stars in the first data release of the SDSS ([6]). We have so far tested twenty-nine DA white dwarf stars near the cool end of the DB gap for pulsational variability in low-degree g-modes, by using the South African Astronomical Observatory (SAAO) 1.9-m telescope ([8]), high-speed photometric observations with the ULTRACAM photometer on the William Herschell 4.2-m telescope and that on the ESO New Technology Telescope ([9]). Among these target stars, we found one of the hydrogen atmosphere DA white dwarfs pulsates in at least one mode with a period of $624 \mathrm{~s}$ ([9]). The amplitude of that mode was near $1 \mathrm{mmag}$ at a $10 \sigma$ confidence level on one night and an $8.4 \sigma$ confidence leveel on a second night. The combined data have a confidence level of $11.8 \sigma$. The VLT UVES spectra clearly shows that the star is a hydrogen atmosphere white dwarf with no trace of helium or metals with $T_{\text {eff }}=32600 \mathrm{~K}, \log g=7.8$ (cgs), and $M=0.55 M_{\odot}$. The star is then consistent with the predicted features of the hot-DAV stars. Besides that, we found two other stars that have significant signals in the period range of g-modes with $\mathrm{S} / \mathrm{N}$ ratios of $6 \sigma$ and $4.4 \sigma$, respectively, on one night, though we did not confirm their pulsation signals on a second night.

The existence of pulsation in this particular DA white dwarf at the cool edge of the DB gap supports the chameleon-like spectral evolution of white dwarfs with thin hydrogen atmosphere. The discovery of further higher amplitude hot-DAV stars would open the possibility of diagnosing of the atmospheric layers of DA white dwarfs in the gap. 


\section{References}

[1] Baglin, A. 1971, in Luyten, W.J. ed, IAU Symp. 42: White Dwarfs. Reidel, Dordrecht, p.136

[2] Eisenstein, D. J. et al. 2006a, ApJS, 167, 40

[3] Eisenstein, D.J. et al. 2006b, AJ, 132, 676

[4] Fontaine, G., Wesemael, F. 1987, in Phillips, A.G.D., Hayes, D.S. Liebert, J. eds, IAU Colloq. 95 : Second Conference on Faint Blue Stars, Davis Press, Schenectady, p.319

[5] Hügelmeyer, S.D., Dreizler, S. 2009, J. Phys. Conf. Ser. 172, 012048

[6] Kleinman, S.J. et al. 2004, ApJ, 607, 42

[7] Kleinman, S.J. 2010, in Werner, K., Rauch, T. eds, 17th European White Dwarf Workshop, American Institute of Physics, AIP Conf. Ser. 1273, p.156

[8] Kurtz, D.W., Shibahashi, H., Dhillon, V.S., Marsh, T.R., Littlefair, S.P. 2008, MNRAS, 389, 1771

[9] Kurtz, D.W., Shibahashi, H., Dhillon, V.S., Marsh, T.R., Littlefair, S.P., Copperwheat, C.M., Gänsicke, B.T., Parsons, S.G. 2012, submitted to MNRAS

[10] Liebert, J. 1986, in Hunger, K., Schönberner, D., Rao, N.K. eds, IAU Colloq. 87: Hydrogen Deficient Stars and Related Objects. Reidel, Dordrecht, p.367

[11] Liebert, J., Wesemael, F., Hansen, C.J., Fontaine, G., Shipman, H.L., Sion, E.M., Winget, D.E., Green, R.F. 1986, ApJ, 309, 241

[12] Shibahashi, H. 2005, in Alecian, G., Richard, O., Vauclair, S. eds, Element Stratification in Stars: 40 Years of Atomic Diffusion, EDP Sciences, Paris, EAS Publications Series, 17, p.143

[13] Shibahashi, H. 2007, in Stancliffe, R.J., Dewi, J., Houdek, G., Martin, R.G., Tout, C.A. eds. Unsolved Problems in Stellar Physics, AIP Conf. Ser. 948, p.35

[14] Unno, W., Osaki, Y., Ando, H., Saio, H., Shibahashi, H. 1989, Nonradial Oscillations of Stars (2nd edition), University of Tokyo Press, Tokyo 